\title{
AVALIAÇÃO DA DISTRIBUIÇÃO ESPACIAL DA TAXA DE ANALFABESTISMO E DE DESEMPREGO NA REGIÃO DO PONTAL DO PARANAPANEMA
}

\section{SPATIAL DISTRIBUTION EVALUATION OF ANALFABESISM AND UNEMPLOYMENT RATE IN THE PONTAL DO PARANAPANEMA REGION}

\author{
Rafael Gonçalves Felippo ${ }^{1}$, Marcos Araujo Lima da Silva ${ }^{1}$, Lucas Prado \\ $\mathrm{Osco}^{2}$, Ana Paula Marques Ramos ${ }^{3}$ \\ Universidade do Oeste Paulista, ${ }^{1}$ Curso de Geografia. ${ }^{2}$ Programa de Pós-Graduação em \\ Agronomia. ${ }^{3}$ Programa de Pós-Graduação em Meio Ambiente e Desenvolvimento \\ Regional - PPGMADRE. Presidente Prudente, SP \\ e-mail: rafaelfelippo@bol.com.br ; marcoskaratebtg@gmail.com,
} anaramos@unoeste.br, pradoosco@gmail.com

RESUMO - O objetivo desse trabalho é realizar a distribuição espacial da taxa de analfabetismo e de desemprego nos municípios do Pontal do Paranapanema para o ano de 2010. O método foi dividido em três etapas: (1) levantamento e organização dos dados referentes à taxa de analfabetismo e de desemprego; (2) preparação da base cartográfica para uso no projeto dos mapas temáticos; e (3) elaboração do mapa de analfabetismo e de desemprego. Tais mapas possibilitaram analisar o padrão na distribuição dos casos de analfabetismo e de desemprego na região do Pontal. Além disso, foi possível identificar quais são os municípios com os maiores problemas de analfabetismo e de desemprego.

Palavras-chave: projeto e produção cartográfica; indicador socioeconômico; Pontal do Paranapanema.

Recebido em: 08/08/2017

Revisado em: 23/08/2017

Aprovado em: 31/08/2017
ABSTRACT - This work aims to perform the spatial distribution of the illiteracy and unemployment rate in the municipalities of Pontal do Paranapanema for 2010. The method was divided into three stages: (1) survey and organization of data on illiteracy rate and unemployment; (2) preparation of the cartographic base to be used in the design of thematic maps; and (3) mapping of illiteracy and unemployment. The maps made it possible to analyze the pattern in the distribution of illiteracy and unemployment in the Pontal region. In addition, it was possible to identify which municipalities have the greatest problems of illiteracy and unemployment.

Keywords: cartographic design and production; socioeconomic indicator; Illiteracy; unemployment; Pontal do Paranapanema. 


\section{INTRODUÇÃO}

O desemprego é uma condição em que pessoas se encontram quando não possuem ou exercem atividades que lhes são rentáveis. Segundo o Instituto Brasileiro de Geografia e Estatística (IBGE, 2016), a taxa de desemprego consiste na percentagem de pessoas desocupadas em relação às pessoas economicamente ativas. De acordo com um levantamento do IBGE, a taxa de desemprego do Brasil atingiu 6,2\% em março de 2015. No Brasil, o desemprego é provocado, dentre outros, pela baixa qualificação, educação precária, trabalhos informais, precariedade na proteção social e êxodo de áreas rurais que são pouco desenvolvidas.

O Brasil possui desigualdades sociais e regionais. Em regiões menos desenvolvidas economicamente se têm os maiores índices de analfabetismo. A região Norte e, principalmente, a Nordeste, apresentam os maiores índices (BRASIL, 2003). No estado de São Paulo, de acordo com o último censo do IBGE, em 2010, há cerca de 1.405.985 pessoas com idade de 15 anos ou mais considerada analfabetas. Uma pessoa é dita analfabeta quando não sabe ler e escrever um bilhete simples no idioma que conhece (IBGE, 2016). A taxa de analfabetismo representa a porcentagem das pessoas analfabetas de um grupo etário, em relação ao total de pessoas do mesmo grupo etário, segundo o IBGE (2016).

O Brasil registrou pouco mais de 13 milhões de pessoas analfabetas, segundo o relatório de 2013 feitos pelo (PNAD) Pesquisa Nacional por Amostra de Domicílios organizada e divulgado pelo Instituto Brasileiro de Geografia e Estatística (IBGE) com base de dados no ano de 2013. Nesse contexto, a realização do presente trabalho se justifica por mostrar a relação entre o analfabetismo e o desemprego no estado de São Paulo, pois, um dos pré-requisitos para muitos dos empregos é, justamente, que o emprego esteja suficientemente alfabetizado.

Tanto o desemprego quanto analfabetismo são temas já discutido por diferentes trabalhos, tais como na obra de Andrade (2011) e Sales e Mathis (2015) sobre desemprego, e Ministério da Educação (2003) e Sguissardi (2011) sobre analfabetismo. A partir do contexto apresentado, o presente estudo revela a necessidade de se investigar a distribuição espacial da taxa de analfabetismo e desemprego na região do Pontal do Paranapanema, região que está localizada à sudeste do Brasil, no extremo oeste do Estado de São Paulo.

A pesquisa se justifica em razão do elevado número de casos de analfabetismo e de desemprego no país e, sobretudo, devido 
à inexistência, até o presente momento, de estudos dessa natureza na região do Pontal. A extensão territorial do Pontal do Paranapanema é $18.844,60 \mathrm{~km}^{2}$ e sua população estimada em 583.703 habitantes, dos quais $89,74 \%$ reside em área urbana, segundo o último Censo do IBGE em 2010. O estado de São Paulo é composto por 15 mesorregiões ou Regiões Administrativas (R.A.) subdivididas em 63 microrregiões ou as chamadas Regiões de Governo. Cada meso e microrregião é formada por um conjunto de municípios e recebe o nome do município sede (IBGE, 2015). O Pontal do Paranapanema pertence à meso e microrregião de Presidente Prudente - SP, sendo essas compostas por 53 e 32 municípios, respectivamente.

O emprego de Geotecnologias vem se destacando no mapeamento de diferentes indicadores sociais (MEDEIROS et al., 2005; MEDEIROS; AlBUQUeRQUE, 2014). A aplicação dos conceitos da Cartografia e a utilização de ferramentas de Geoprocessamento, por meio de software de Sistema de Informação Geográfica (SIG), poderão facilitar a investigação da distribuição da taxa de analfabetismo e de desemprego nos diferentes municípios do Pontal do Paranapanema. Isso porque possibilita a análise da taxa desses indicadores em função da localização geográfica dos municípios e, também, viabiliza a comparação entre distintos municípios. Sendo assim, este trabalho tem por objetivo realizar o mapeamento da distribuição da taxa de analfabetismo e de desemprego na região do Pontal do Paranapanema no ano de 2010.

\section{METODOLOGIA}

Materiais

No desenvolvimento da pesquisa foram utilizados computador e impressora, software de SIG, base cartográfica dos municípios do estado de São Paulo disponível no banco de dados do IBGE, e dados da taxa de analfabetismo e de desemprego disponíveis no banco do Ministério da Saúde (DATASUS)

\section{Método}

O método foi dividido em três etapas: (1) levantamento e organização dos dados referentes à taxa de analfabetismo e de desemprego; (2) preparação da base cartográfica para utilizar no projeto dos mapas temáticos; e (3) elaboração do mapa de analfabetismo e de desemprego.

Levantamento e organização dos dados

$$
\text { O levantamento dos dados de }
$$
analfabetismo e do desemprego foi realizado no sistema online do DATASUS (http://datasus.saude.gov.br/). Na página inicial, seleciona-se a opção Acesso à Informação e, em seguida, é possível 
escolher os censos a serem pesquisados. Para a taxa de analfabetismo foi utilizado o censo de educação do ano de 2010, utilizando-se a base territorial em nível municipal. Para taxa de desemprego de pessoas com 15 anos ou mais foi usado o censo de trabalho e renda de 2010 e base territorial em nível municipal Microrregião IBGE/Pres. Prudente.

Os dados serão organizados no Microsoft Excel. A taxa de analfabetismo é calculada de acordo com os critérios do indicador de Taxa de alfabetização, dos Indicadores e Dados Básicos da Rede Interagencial de Informações para a Saúde (RIPSA, 2008). O cálculo é realizado considerando o número de pessoas residentes de 15 e mais anos de idade, por grupo de anos de estudo, dividido pela população total residente desta faixa etária multiplicado por 100. A taxa de desemprego é calculada segundo os critérios do indicador de Taxa de desemprego, dos Indicadores e Dados Básicos da RIPSA (2008). O cálculo considera o número de residentes de $10 \mathrm{e}$ mais anos de idade que se encontram sem trabalho, em determinado espaço geográfico, no ano considerado, dividido pelo número de residentes economicamente ativos desta faixa etária multiplicado por 100.

Preparação da Base Cartográfica

A base cartográfica contendo o limite dos municípios do Estado de São Paulo foi obtida no banco de dados cartográficos do IBGE. A partir disso, aplicou-se ferramentas de Geoprocessamento, usando o software de SIG, o QGIS, para editar a base de modo a conter apenas os 32 municípios que compõe o Pontal do Paranapanema. Um total de 2 mapas coropléticos foi elaborado, um para representar a distribuição espacial da taxa de analfabetismo e outro para a taxa de desemprego.

\section{RESULTADOS}

$\mathrm{Na}$ Figura 1 abaixo está à representação da taxa de analfabetismo em 2010 nos 32 municípios do Pontal do Pontal do Paranapanema. Observou-se que a cidade de Presidente Prudente possui o menor número de casos de analfabetos na região, sendo que a cidade de Euclides da Cunha, Sandovalina, Piquerobi, Caiabu e Nantes, possuem os maiores índices. 
Figura 1. Mapa da distribuição da taxa de analfabetismo no Pontal do Paranapanema - SP em 2010.

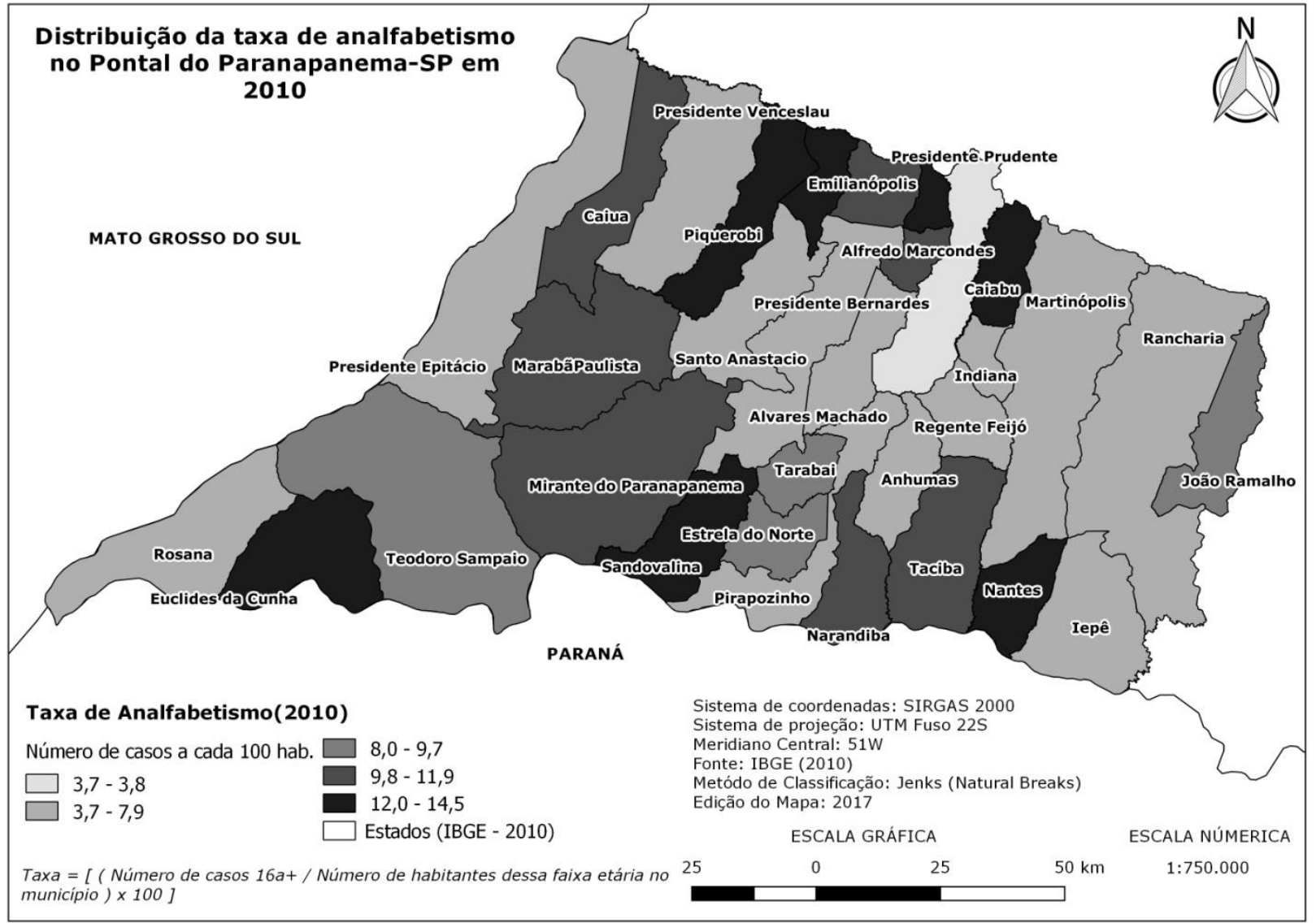

$\mathrm{Na}$ Figura 2 a seguir tem-se à representação espacial da taxa de desemprego em 2010 nos 32 municípios do Pontal do Pontal do Paranapanema. Compreendeu-se que os municípios com maior número de desempregados foram: Presidente Epitácio, Rosana, Euclides da Cunha, Teodoro Sampaio, Estrela do Norte, Tarabai, Indiana e Narandiaba. Sendo Anhumas a cidade com menor número de desempregados.

Figura 2. Mapa da distribuição da taxa de desemprego no Pontal do Paranapanema - SP em 2010 


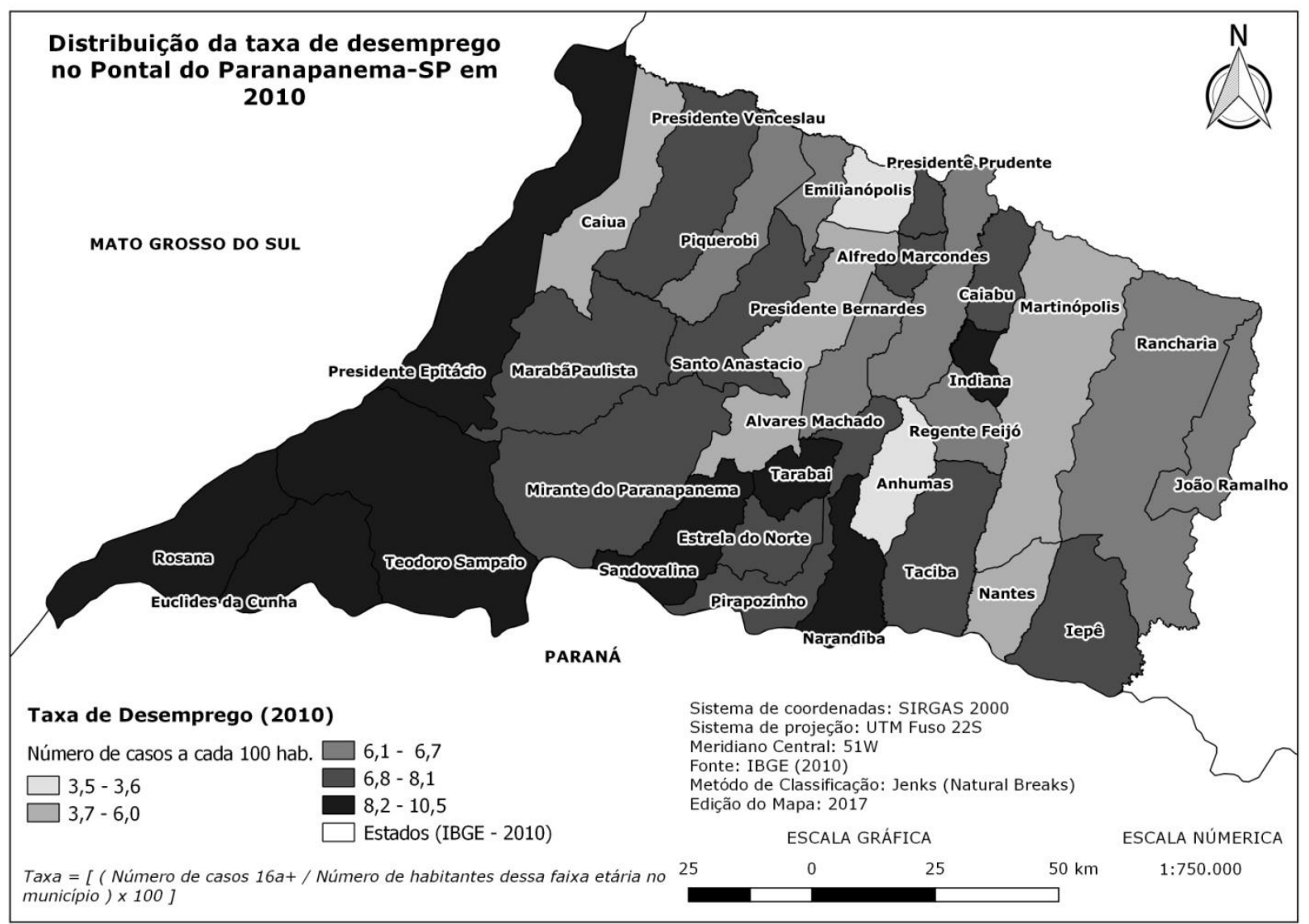

\section{CONCLUSÃO}

Para a elaboração dos mapas de distribuição espacial da taxa de analfabetismo e de desemprego na região do Pontal do Paranapanema, realizou-se a coleta de dados no IBGE e no DATASUS onde foi possível um levantamento inicial para a formulação de uma base cartográfica por meio de um software de SIG. A partir da espacialização dos dados, identificou-se os municípios em que ocorrem com maior frequência os fenômenos de desemprego e analfabetismo nessa região.

Conclui-se que na região do Pontal do Paranapanema há um analfabetismo em pequena proporção. Todavia, o mais preocupante é a relação de desemprego na região que supera a taxa de analfabetos. Entende-se que a alta taxa de desemprego não é uma consequência apenas da ausência de mão-de-obra qualificada, mas sim possivelmente provocada por outros problemas. Futuras investigações possam ser realizadas, especificamente nos municípios com os piores índices, a fim de compreender os possíveis fatores que contribuíram para elevadas taxas nesses locais. 


\section{REFERÊNCIAS}

ANDRADE, P. C. R. Estudo da série da taxa de desemprego na região metropolitana de Recife. Revista da Universidade Vale do Rio Verde. v 9. n. 1, p. 3-18. 2011.

BRASIL. Ministério da Educação. Instituto Nacional de Estudos e Pesquisas Educacionais Anísio Teixeira (INEP). Mapa do Analfabetismo no Brasil (2003). 2003. Disponível em: <http://www.oei.es/quipu/brasil/estadisticas/an alfabetismo2003.pdf> Acesso em: 13 abr. 2016.

IBGE. Instituto Brasileiro de Geografia e Estatística. Conceitos. 2016. Disponível em:

<http://www.ibge.gov.br/home/estatistica/popul acao/condicaodevida/indicadoresminimos/concei tos.shtm> Acesso em: 13 de abril de 2016.

MEDEIROS, C. N.; PETTA, R. A.; DUARTE, C. R. Mapeamento de indicadores socioeconômicos do município de Parnamirim (RN) utilizando técnicas de Geoprocessamento. Revista Geografia. v 30. n. 3, p. 507-524. 2005.

MEDEIROS, C. N.; AlBUQUeRQUE, E. L. S. Mapeamento da vulnerabilidade social em nível de setores censitários: estudo de caso para o município de Caucaia (CE). IPECE.v 1. n. 107, p. 121. 2014.

RIPSA - Rede Interagencial de Informações para a Saúde. Indicadores e Dados. Básicos. 2008. Disponível em <http://tabnet.datasus.gov.br/tabdata/livroidb/2 ed/indicadores.pdf $>$. Acesso em 08 de junho de 2017.

SALES, C. R. L.; MATHIS, A. A. Desemprego e qualificação profissional na região de CarajásPará. Argumentum. v 7. n. 1, p. 184-201. 2015.

SGUISSARDI, V. Decifrando a história e o estigma do analfabetismo no Brasil. Educ. Soc.v 32. n. 114, p. 245-249. 2011. 